\title{
Prácticas en búsqueda de legitimidad: el uso contemporáneo de la ayahuasca, entre reivindicaciones terapéuticas y religiosas
}

\author{
Practices in search of legitimacy: the contemporary \\ use of ayahuasca, between religious and therapeutic \\ vindications
}

${ }^{1}$ Doctor en Antropología Social. Investigador posdoctoral, University of Durham, Durham, United Kingdom. $\triangle$ (iD)
RESUMEN El creciente interés de los occidentales por la bebida psicotrópica ayahuasca y la participacion en rituales exóticos ha llevado en las últimas décadas a la multiplicación de los "centros chamánicos" en la Amazonía peruana. Entre estos, el centro Takiwasi es una comunidad terapéutica que acoge cada año a cientos de clientes nacionales y extranjeros. Esta institución, creada por un médico francés en 1992, fue originalmente destinada a proponer una alternativa terapéutica para el tratamiento de la adicción, y se caracterizaba por el uso de ciertas herramientas del chamanismo mestizo peruano articuladas con la biomedicina y la psicología clínica. La evolución diacrónica de la institución está, sin embargo, marcada por la creciente utilización de elementos de la tradición católica. Examino aquí la hipótesis de que estas transformaciones pueden ser interpretadas como los efectos de la globalización del uso de la ayahuasca y sus consecuencias jurídicas y políticas. De este modo, el caso de Takiwasi subraya el papel jugado por las tradiciones religiosas y el campo médico en la construcción, la legitimación y el mantenimiento de las prácticas nuevas e híbridas que se multiplican alrededor del uso de la ayahuasca.

PALABRAS ClAVES Religión; Chamanismo; Catolicismo; Perú.

\begin{abstract}
In recent decades, the growing interest of Westerners in the psychotropic brew ayahuasca and the participation in exotic rituals has led to the multiplication of "shamanic centers" in the Peruvian Amazon. Among these, Takiwasi is a therapeutic community that welcomes hundreds of national and foreign clients every year. This institution, created by a French physician in 1992, was originally intended to propose a therapeutic alternative for the treatment of addiction, characterized by the use of tools of Peruvian mestizo shamanism, biomedicine and clinical psychology. The diachronic evolution of the institution is however marked by the growing use of elements of the Catholic tradition. In this article, I will examine the hypothesis that these transformations can be interpreted as the effects of the globalization of the use of ayahuasca and its legal and political consequences. Thus, the case of Takiwasi underlines the role played by religious traditions and the medical field in the construction, legitimization and maintenance of new and hybrid practices that are multiplying around the use of ayahuasca.
\end{abstract}

KEY WORDS Religion; Shamanism; Catholicism; Peru. 


\section{INTRODUCCIÓN}

La participación en rituales exóticos percibidos como tradicionales y vividos como prácticas religiosas, terapéuticas o de desarrollo personal tiene un éxito muy importante entre el público occidental desde la segunda mitad del siglo XX. Alimentados en el interés por el brebaje psicotrópico ayahuasca (Banisteriopsis caapi), así como la imagen mítica de la figura del chamán y de la selva "primigenia", un flujo de viajeros se mueve hacia la Amazonía peruana desde la década de 1990.

Durante los últimos veinte años, muchos lugares de acogida para esta nueva clientela aparecieron en el borde de las ciudades de la Amazonia peruana (lquitos, Pucallpa, Tarapoto). Estas instituciones, llamadas por AnneMarie Losonczy y Silvia Mesturini Cappo ${ }^{(1)}$ como "centros chamánicos", se basan frecuentemente en la asociación entre europeros y mestizos locales o indígenas, y ofrecen actividades rituales presentadas como inspiradas en la "medicina tradicional amazónica". Articulando elementos discursivos y pragmáticos de muy distintos orígenes culturales, los dispositivos ofrecidos por estas instituciones se basan más o menos libremente en las prácticas de los curanderos mestizos y urbanos de la Amazonia peruana que cubren múltiples especializaciones (hierbas, perfumes, oración, parto y alucinógenos) $)^{(2)}$.

Fundada en 1992 por el médico francés Jacques Mabit, en colaboración con tres peruanos y una española, la comunidad terapéutica Takiwasi es, a la vez, una clínica de tratamiento de adicciones y uno de los principales lugares que ofrecen sus servicios a estos viajeros extranjeros en el Amazonas peruano. La institución ha desarrollado un dispositivo terapéutico que se caracteriza por la recuperación de elementos de la farmacopea nativa (quechua lamista) tales como plantas eméticas o ayahuasca. Esta recuperación se acompaña de una profunda transformación de las representaciones taxonómicas, así como de la teoría etiológica y terapéutica a la que se ajusta al uso de esas plantas ${ }^{(3)}$.
Si el proyecto de Takiwasi fue originalmente destinado a proporcionar una alternativa terapéutica a la adicción a las drogas mediante la combinación de la biomedicina y la "medicina tradicional amazónica", la influencia de los elementos de la corriente New Age y luego del catolicismo, fue creciendo en la historia de la institución, lo que distingue ahora Takiwasi de los numerosos centros chamánicos en la región. ¿Qué nos dice esta transformación de la institución y del contexto social de su desarrollo?

He mostrado en otros escritos ${ }^{(3)}$ que estas transformaciones, desde un punto de vista emic, se enraízan en la experiencia ritual de los oficiantes y en las operaciones de socialización de las alucinaciones inducidas por la ayahuasca. En este artículo, voy a examinar la hipótesis de que estas transformaciones pueden ser interpretadas como los efectos de la globalización del uso de la ayahuasca y sus consecuencias jurídicas y políticas. En este sentido, las transformaciones de Takiwasi podrán ser leídas como estrategias de legitimación en un contexto de estigmatización del uso de la ayahuasca. De este modo, el caso de Takiwasi nos permite entender el papel jugado por las tradiciones religiosas y el campo médico en la construcción, la legitimación y el mantenimiento de las prácticas nuevas e híbridas que se multiplican alrededor del uso de la ayahuasca en las últimas décadas.

\section{ASPECTOS METODOLÓGICOS}

Para desarrollar los puntos mencionados, voy a apoyarme en el análisis de la comunicación pública de la institución (publicaciones de artículos, conferencias, documentales, páginas web, etc.), así como en los datos recogidos durante un trabajo de campo etnográfico realizado durante un período de 18 meses dividido en tres estancias de investigación, entre 2008 y 2013.

La investigación se llevó a cabo en la región de San Martín (Alta Amazonía del Perú), la mayor parte del tiempo en Takiwasi. En 2009, se realizó una primera estancia de 
cuatro meses, en la que me centré en el tratamiento de las adicciones propuesto en Takiwasi, usé diferentes modos de recopilación de datos, entre los cuales el estudio de las interacciones cotidianas y rituales a través de la observación de los participantes desempeñó un papel central, junto con entrevistas e historias de vida de unos diez pacientes drogodependientes y de los principales actores de la institución (especialistas rituales y psicólogos).

En 2011, realicé una segunda estancia de seis meses, en la que frecuenté cuatro "seminarios" ofrecidos a clientes extranjeros, en cada uno de los cuales participaron entre 15 y 20 personas. Compartí las actividades diarias y rituales de los participantes, así como los grupos de palabra. Realicé también entrevistas individuales e historias de vida con alrededor de treinta participantes.

En 2013 se llevó a cabo una visita final de ocho meses. En esta ocasión, presencié tres nuevos "seminarios", con un total de cuarenta y cinco participantes. También realicé entrevistas semanales con dos pacientes drogadictos internados durante seis meses y, más episódicamente, con otros pacientes. Esta investigación también se complementó con un estudio comparativo, cuyo objetivo era conocer mejor las especificidades de la institución estudiada en relación con las prácticas del curanderismo mestizo o lamista de la región.

Esta investigación etnográfica -que en Francia no necesita la aprobación de un comité de ética- se basó en el respeto del "consentimiento informado" y fue realizada con participantes voluntarios que conocían los objetivos del estudio y que sabían que podían abandonar la investigación si así lo deseaban.

\section{RESULTADOS}

\section{La fundación de un centro de tratamiento para los adictos a las drogas y de investigación sobre las medicinas tradicionales: historia de Takiwasi}

La historia de Takiwasi se enraíza en la de sus fundadores, entre ellos, Jacques Mabit, quien actualmente es la figura central de la institución. Aquí proponemos una síntesis de los trabajos académicos que han descrito la historia de Takiwasi ${ }^{(4,5,6)}$ y entrevistas con Jacques Mabit y con diversos actores involucrados en la historia de la institución, incluyendo empleados (curas, psicólogos, etc.) y algunos de sus cofundadores.

Después de obtener un doctorado en Medicina por la Université de Nantes, en 1984, Jacques Mabit se suma a misiones breves en diferentes organizaciones no gubernamentales (ONG) francesas, incluyendo misiones en Perú. Estas experiencias dan lugar al proyecto para llevar a cabo una investigación sobre la "medicina tradicional". Siguiendo el consejo de un amigo, se fue al Alto Amazonas, al departamento de San Martín, para encontrar curanderos. Y allí, en la ciudad de Tarapoto, en la casa de Rosa Giove, quien se convertiría unos años más tarde en su esposa, Jacques Mabit encuentra a José Campos, un peruano nativo de Cajamarca. Jacques Mabit comienza entonces un trabajo de investigación sobre "sistemas mentales de representación de las prácticas de atención en el departamento de San Martín"(7), y adopta un método que se caracteriza por la "autoexperimentación" de las prácticas estudiadas ${ }^{(7)}$. Entre 1986 y 1993 se encuentran con alrededor de setenta curanderos de la región, mestizos o lamistas, que lo introducen al uso de plantas medicinales, canciones rituales (icaros) y técnicas tales como la soplada (que consiste en soplar el humo del tabaco o perfumes líquidos sobre el cuerpo del paciente para su purificación y protección). Jacques Mabit y José Campos llegan luego a conocer a Roni Yon, peruano de nacimiento, de la Sierra de Huamachuco, recientemente emigrado a Tarapoto. Algún tiempo después, el grupo encuentra a Dionísio Santos, franco-español establecido hacía diez años en Perú. Profesor en la Alianza Francesa, había conocido a Don Solón Tello Lozano, un curandero mestizo que lo había iniciado en las prácticas del vegetalismo y el uso de plantas medicinales, incluyendo tabaco y alucinógenos tales como la ayahuasca. Así se forma el grupo detrás de la fundación de Takiwasi. 
Líder mundial en la producción de coca, la región de San Martín, se enfrenta en este periodo a un fuerte consumo local de pasta base de cocaína. A pesar de la creciente demanda de atención, los lugares de tratamiento son pocos y están concentrados en la capital. La región es una de las zonas más pobres del país, afectada por la presencia de traficantes de drogas y la violencia relacionada con el conflicto armado entre la guerrilla de Sendero Luminoso y el Movimiento Revolucionario Túpac Amaru (MRTA). En este contexto, Jacques Mabit pretende ofrecer una alternativa terapéutica para tratar la drogadicción movilizando recursos locales.

En 1990, dirigió con este propósito una misión para estudiar las técnicas de tratamiento de drogas en el contexto de las "medicinas tradicionales" locales en las Filipinas y Tailandia. En agosto de 1992 la ONG Takiwasi es registrada como una asociación sin fines de lucro por Jacques Mabit, Rosa Giove, José Campos, Rony Yon Dionísio Santos en el municipio de Tarapoto. El "centro de rehabilitación para adictos a las drogas y de investigación en las medicinas tradicionales Takiwasi" se abre en septiembre y recibe rápidamente el permiso del Estado peruano para ejercer como centro de tratamiento de la adicción.

Los principales actores de Takiwasi desarrollan una política de comunicación basada, en particular, en la publicación anual de una revista entre 1992 y 1997, artículos ${ }^{(8)}$, libros $^{(9)}$ y conferencias dirigidas por Jacques Mabit en todo el mundo. Eso garantiza la visibilidad de la institución, que se presenta como un centro de investigación pionero en la medicina tradicional amazónica y su aplicación en el tratamiento de las adicciones.

El financiamiento de donantes privados, del gobierno francés (1992-1994), de la Comunidad Europea (1993-1995) y de las Naciones Unidas (1995-1997) permite la adquisición de tierras, construcción de infraestructura y la remuneración de algunos empleados. Rony Yon está entonces a cargo de la logística y el desarrollo de las preparaciones de hierbas, Jacques Mabit, José Campos y Dionísio Santos lideran celebración de rituales. Si la base de Takiwasi es el trabajo de varios individuos, las diferencias personales llevaron gradualmente a los cofundadores a abandonar la dirección de Takiwasi y dejarla en manos de Jacques Mabit. Hoy en día, solo Rosa Giove, esposa de Jacques Mabit, ejerce una actividad dentro de la institución. Rony Yon, Jose Campos y Dionísio Santos establecieron sus propias instituciones que ofrecen servicios (ritual de purga, ritual de ayahuasca, dieta) para los clientes extranjeros.

Por lo tanto, Jacques Mabit es ahora la principal autoridad de Takiwasi. Todas las decisiones importantes relativas a las actividades de la institución están sujetas a su aprobación. El médico francés es también el principal personaje público de Takiwasi, y asegura la comunicación internacional de la institución a través de conferencias, artículos o documentales. Si este último es el titular principal de la autoridad ritual, otras personas tienen derecho a oficiar durante los rituales ofrecidos por la institución, como Rosa Giove, o Jaime Torrès, psicólogo clínico que se convirtió en director de Takiwasi. Desde su creación, Takiwasi también emplea mestizos y curanderos indígenas para contribuir al logro de las prácticas rituales. El establecimiento cuenta con cuarenta empleados. La mayoría de ellos son originarios de la región, y se dedican a tareas de mantenimiento, gestión y administración de la institución. Un grupo de seis personas se dedican a la producción y comercialización de hierbas medicinales en el laboratorio. Un equipo que incluye médicos, psicólogos y especialistas rituales ofrece servicios de tratamiento.

En veinte años, Takiwasi se transforma en una clínica que recibe permanentemente a quince pacientes y residentes, y que publicita el tratamiento de más de quinientos adictos a las drogas. El tratamiento de la adicción propuesto por Takiwasi consiste en un proceso de nueve meses. En el modelo de comunidades terapéuticas, quince pacientes comparten la vida diaria y residen en dormitorios. Por la mañana, los pacientes realizan diversas tareas (limpieza, jardinería, cocina) y asisten a talleres durante la tarde (deportes, manualidades, arte). Cada residente es 
seguido por un psicoterapeuta acreditado que se reúne semanalmente para una entrevista. En 2014, el equipo estaba formado por cuatro psicólogos (de nacionalidad francesa, checa, española y colombiana). La participación en las prácticas rituales que acompaña a la ingestión de plantas medicinales es obligatoria: rituales de purga, de ayahuasca semanales y aislamiento ("dieta") durante diez días cada dos meses.

Más allá de sus actividades clínicas, la asociación también tiene como objetivo participar en la valorización de la "medicina tradicional" en la región, con el fin de promover su legitimidad frente a los órganos políticos y el mundo científico. Uno de los principales resultados de estas aspiraciones fue la asignación del estatuto de "patrimonio nacional y cultural" de Perú a la ayahuasca, en 2008, por parte del Ministerio de Cultura. En efecto, la propuesta de esta declaración había sido redactado y apoyada por Rosa Giove, esposa de Jacques Mabit y cofundadora de Takiwasi.

La institución se ha comprometido también a la producción de datos clínicos para certificar los resultados de los métodos de la "medicina tradicional amazónica" en el tratamiento de adicciones ${ }^{(9)}$ y psicopatologías. Para dar mayor visibilidad a estos proyectos, Takiwasi organizó varias reuniones internacionales tal que el Foro Internacional del Consejo Indígena Interamericano Espiritualidad Sober, en 1998, y el Congreso Internacional "Medicinas, Interculturalidad y Salud Mental", en 2009.

\section{Teoría etiológica y terapéutica de la adicción}

Durante los años en que seguí la creación de Takiwasi, los principales actores de la institución publican varios artículos en los que, progresivamente, formulan una teoría original de la adicción. Según estos textos, las adicciones plantean la cuestión de "la aspiración del individuo a lo sagrado y la espiritualidad" a través de un "camino iniciático" que no se puede encontrar en las sociedades "modernas" debido a su secularización ${ }^{(8)}$. En esta perspectiva, la adicción sería el resultado de una aspiración espiritual lograda a través de una autoiniciación que, carente de estructura social, produciría una "contrainiciación", conduciendo a la persona a la autodestrucción y la marginación. El tratamiento de la adicción debería así lograr una "verdadera iniciación" estructurada a través de los recursos de la "medicina tradicional amazónica"(8). En este sentido, la adicción se entiende aquí como el resultado de una búsqueda espiritual, por lo que la curación se asocia con la búsqueda de una experiencia directa de la trascendencia asociada a la experiencia de "estados alterados de conciencia" por el uso ritual de sustancias psicotrópicas como la ayahuasca ${ }^{(8)}$.

Se reconoce aquí la influencia de la psicología transpersonal, que domina el paradigma psicoterapéutico en Takiwasi. El término transpersonal significa "más allá" de lo personal y se refiere a las experiencias, procesos y eventos que, según los principales representantes de este corriente (Roberto Assagioli, Abraham Maslow, Stanislav Grof), transcienden la habitual sensación de identidad, permitiendo experimentar una realidad mayor y más significativa ${ }^{(10)}$.

El uso de la ayahuasca en este contexto está también influenciado por la valoración del uso psicoterapéutico de alucinógenos, defendido por el movimiento psicodélico e iniciado por las experiencias de "terapiaLSD", dirigida por Stanislav Grof ${ }^{(11)}$. Se asigna a la ayahuasca una función de catalizador de la psicoterapia (facilitación de las asociaciones verbales, generación de imágenes mentales, abreacción, regresiones infantiles). Las propiedades de la ayahuasca son, en este sentido, vistas como útiles por un proceso psicoterapéutico, si la experiencia alucinógena es seguida de un espacio de desarrollo verbal. Esta es la razón por la cual, en Takiwasi, los rituales de ayahuasca se articulan con espacios de reflexión para aclarar la experiencia ritual del participante, en el que se proponen entrevistas individuales durante las cuales el paciente cuenta la historia de su experiencia a un psicoterapeuta, cuya función es ayudar en su interpretación. Los grupos de discusión también se organizan para evocar 
la experiencia ritual: se reúnen a todos los pacientes y se llevan a cabo rituales conducidos por los psicólogos o especialistas. Como una prueba proyectiva, el ritual de la ayahuasca, en este contexto, es diseñado como una herramienta para expresar las disposiciones psicológicas inconscientes del participante. Así, se establece un paralelo entre la experiencia ritual del participante y su vida cotidiana. Mediante la articulación de los diferentes aspectos de la narración del participante con su historia personal, su economía relacional y sus disposiciones psicológicas, estas interacciones discursivas invitan a los participantes a utilizar su experiencia ritual como una herramienta heurística que le permite el acceso a una mejor comprensión de sí mismo como sujeto psicológico. Las técnicas rituales que regulan el uso de ayahuasca tienen así la función del setting de las terapias psicodélicas ${ }^{(12)}$ : un dispositivo de contención simbólica que acompaña la absorción de alucinógenos. Así se intenta evitar problemas potenciales en el participante y garantizar la "estructura simbólica" de la experiencia.

Durante nuestras conversaciones con los psicoterapeutas de Takiwasi, ellos destacaron que muchas de las experiencias que surgen en el ritual de la ayahuasca serían, sin embargo, incomprensibles a la luz de los datos biográficos del individuo, y que esta experiencia revelaba elementos "transpersonales", relacionados con lo transgeneracional (síntomas o patologías heredados de un antepasado), lo perinatal (síntomas o patologías relacionados con los eventos que ocurrieron durante la vida intrauterina) o la relación de la persona con algunos seres sobrenaturales. En este sentido, el uso de la ayahuasca sería, según ellos, una herramienta terapéutica valiosa: permitiría el acceso a materiales perinatales, transgeneracionales, y mostraría relaciones conflictivas del paciente con seres espirituales; permitiendo así curar con el tiempo los síntomas asociados con estos elementos.

\section{¿Surgimiento de un movimiento religioso transnacional?: la influencia de la religiosidad contemporánea occidental}

En contradicción con el enfoque biomédico de la psicopatología, esta teoría etiológica y terapéutica revela la influencia de las teorías de Jung y de las corrientes de psicoterapia desarrolladas en el contexto de la contracultura y del surgimiento de la New Age ${ }^{(13)}$. Los artículos publicados por Takiwasi revelan así la influencia de las ideas propias de la "nebulosa psicomística"(14), como la convergencia armoniosa entre la ciencia moderna y los "conocimientos tradicionales". El proyecto de Takiwasi se revela también como parte del movimiento "neochamánico"(15), marcado por la universalización del chamanismo y la extrapolación de su contexto original, o la aproximación de las prácticas chamánicas con técnicas psicoterapéuticas y desarrollo el personal.

Además de la comunicación producida por la institución, Takiwasi ha sido objeto de numerosas publicaciones -artículos de investigación académica ${ }^{(4,6,16)}$, , así como documentales radiales ${ }^{(17)}$ o audiovisuales ${ }^{(18,19)}$. Estos medios de comunicación transmiten una imagen de la institución que cristaliza de nuevo ciertos ideales de la religiosidad occidental contemporánea de la Nueva Era y de la "cultura neochamánica"(4). Los artículos, documentales, conferencias, etc., dan una importancia central a la biografía de Jacques Mabit, y aquellos documentos que cuentan su historia de vida y la creación de Takiwasi desarrollan "una especie de mito en torno a la figura de Jacques Mabit alimentando gradualmente los imaginarios de las personas que vienen para encontrarse con él o hacer juicios en su contra"(16).

Jacques Mabit comienza la historia de su itinerario biográfico evocando una "crisis" ocurrida en 1984 a la edad de 28 años, alimentada por "cuestiones existenciales, problemas personales, relacionales y psicológicos $^{\prime \prime(20)}$. En este contexto se va a Calcuta para encontrar a la Madre Teresa. En India, vive una "experiencia de la muerte" que lo impulsa 
a regresar a Perú para tratarse con los "curanderos" que había conocido durante sus visitas anteriores. En esta época participa por primera vez de un ritual de ayahuasca. Así comenzó lo que se describe en un documental ${ }^{(18)}$ como un "camino iniciático" con curanderos de San Martín, que encuentra su expresión en la asignación de una misión:

El centro Takiwasi, que es una realidad física, material y razonable, [...] surge de una información que es irracional, porque proviene de una sesión de ayahuasca; con una visión en la que vi personajes -que eran una especie de jurado- que me dijo "somos los espíritus guardianes de la selva". Y me preguntaron lo que quería, por qué estaba frente a ellos. Le dije: "quiero aprender esta medicina". Se consultaron y el que estaba en el centro dijo: "bueno, está bien, se le permite entrar en este territorio, pero va a tener que hacer eso". Y allí me vi a mí mismo tratar a los adictos. ${ }^{(18)}$

En esta época, a Jacques Mabit le parece que el trabajo con adictos es difícil y no se ajusta a su "misión". Sin embargo, continúa su "iniciación" a través de la participación en rituales de ayahuasca y el aislamiento de las "dietas". En 1989, una nueva visión durante un ritual de ayahuasca le recuerda su misión:

Después de tres años, tuve de nuevo una visión. Una mujer apareció y me dijo «hay que empezar a trabajar con adictos a las drogas». Luego trató de negociar. Ella dijo que cuando un niño nace, tiene un tiempo de gestación para prepararse, pero después, hay que nacer. ${ }^{(18)}$

Jacques Mabit comienza a viajar por Europa, Asia y América del Norte, para buscar fondos para realizar su proyecto y, luego, aparece como guiado en esta misión a través de sueños y "sincronicidades", que lo llevan a fundar Takiwasi.

La biografía de Jacques Mabit, como se presenta en estos documentos, evoca sorprendentemente el "viaje interior del New
Age $^{\prime \prime(13)}$. Un choque inicial provoca en Jacques Mabit una búsqueda que lleva a la operación de transformación, que lo llevará a una revelación personal y a la adjudicación de una misión de inspiración sobrenatural. Esta biografía de un "doctor convertido en chamán" cristaliza mitemas ${ }^{(21)}$ específicos de las historias de iniciación que componen la literatura de la New Age ${ }^{(16)}$. La encarnación de estos elementos de la religiosidad occidental contemporánea de inspiración New Age por parte de Jacques Mabit parece contribuir al poder de atracción de Takiwasi después de la audiencia occidental. Estos atributos dan a Jacques Mabit una autoridad carismática que Del Bosque relaciona con el carisma profético $^{(4,22,23)}$.

\section{El giro católico de la institución}

Si bien el proyecto inicial de Takiwasi fue fuertemente influenciado por la religiosidad contemporánea occidental de la corriente New Age, el peso de las representaciones católicas parece haber sido cada vez mayor en la historia de la institución. En 2004, se organizaron en Takiwasi una serie de conferencias tituladas "encuentro entre el cristianismo y el chamanismo", a las que asistieron veinte participantes peruanos y franceses, miembros de la Iglesia católica y de Takiwasi, para desarrollar una reflexión sobre las actividades de la institución ${ }^{(4)}$. Durante esta conferencia, los principales actores Takiwasi cuestionan el "turismo chamánico" emergente, y lo interpretan como la manifestación de una búsqueda espiritual favorecida por la secularización de las sociedades occidentales. Son denunciados los malentendidos del neochamanismo nacidos de la idealización de la figura del chamán en las sociedades occidentales. Condenando la tendencia al sincretismo espiritual y las espiritualidades del tipo New Age, los actores Takiwasi anclan su práctica en la institución católica. Sobre la base de diversas fuentes de la tradición católica, las partes interesadas trabajan para registrar las prácticas Takiwasi en línea con la doctrina de la Iglesia. En esta perspectiva, el uso ritual de la ayahuasca es presentado por primera vez como un vector 
potencial de la evangelización del público occidental. Jacques Mabit Ilama la atención sobre los fenómenos de posesión e infestación demoníacas que, según él, afectan a los clientes de la institución, y que él considera como infravalorados por la Iglesia.

El equipo de Takiwasi formula entonces un cuerpo original de representaciones cosmológicas y etiológicas, que lo distinguen del curanderismo peruano y del turismo chamánico (religiosidad New Age, desarrollo personal, etc.), y el concepto de la infestación es el centro de esta teoría. Tomado de la teología católica, este concepto designa inicialmente una modalidad de la influencia demoníaca menor y más común que la posesión, caracterizada por la presencia de una entidad demoníaca que afecta a la persona en su salud, su fe o sus pensamientos ${ }^{(24)}$. En Takiwasi, la infestación se describe como una relación de tipo parasitaria mantenida con uno o varios seres sobrenaturales demoníacos. Esta condición, que se describe como causa de los trastornos físicos y psicológicos, se concibe como el resultado de la transgresión de tabúes (consumo de drogas, sexualidad, prácticas mágicas, espiritismo, etc.), el contacto con lugares o personas, o debido a la transmisión a través de la paternidad. Este mal requiere de un tratamiento específico, que consiste en la purificación del cuerpo a través de la absorción de preparaciones eméticas, ayahuasca y prácticas propuestas por la Iglesia católica, como oraciones de exorcismo. El ritual de ayahuasca, en este contexto, es considerado como una herramienta para detectar y tratar fenómenos de infestación ${ }^{(25)}$.

El mismo año, el obispo de Moyobamba, a petición de Jacques Mabit, consagra la capilla de Takiwasi y nombra al padre Christian Alejandria como responsable. A petición de la institución, en 1990, el sacerdote es mandatado por el obispado para proporcionar acompañamiento pastoral al personal y a los pacientes de Takiwasi. En esta época se producen una serie de apariciones de la Virgen a miembros o clientes de Takiwasi a través de los sueños y visiones de ayahuasca, que dan lugar a la construcción de varios lugares u objetos de culto católicos en los terrenos de
Takiwasi, como la imagen de la Virgen de la Puerta o la Cueva de la Virgen. Varios lugares de culto católicos fueron construidos en Takiwasi en los años siguientes, como la capilla dedicada a San Martín de Porres, construida siguiendo una visión de Jacques Mabit recibida durante un ritual de ayahuasca. La creciente influencia de las representaciones católicas se ve también en las prácticas rituales, el uso de oraciones de exorcismo, del crucifijo, del agua bendita o por la inserción de las principales figuras del panteón católico en los cantos rituales y el rango de los espíritus auxiliares de los especialistas rituales (Cristo, la Virgen María, los santos y los ángeles).

Estos cambios nos invitan a cuestionar la dimensión religiosa de una institución que se presenta públicamente como un "centro de tratamiento de la adicción" al "encuentro entre la medicina occidental y la medicina tradicional amazónica". Además, algunos años después de la fundación de Takiwasi, antiguos clientes propagan en Francia prácticas inspiradas en el dispositivo de Takiwasi, que moviliza el cuerpo de representaciones cosmológicas, etiológicas y terapéuticas de la institución. Dirigido por personas que participaron regularmente en las prácticas rituales de Takiwasi y autorizados por Jacques Mabit para impulsar estas prácticas, dos asociaciones y una escuela privada de psicoterapia pasan a ser los principales asociados de la institución de Perú en Francia.

La formación de un cuerpo de especialistas capaces de exportar las prácticas rituales llevó a ciertos observadores ${ }^{(4)}$ a pensar a Takiwasi en términos de movimiento transnacional terapéutico-religioso en proceso de constitución ${ }^{(26)}$. En efecto, Takiwasi presenta una forma de organización social que evoca a los movimientos religiosos en proceso de transnacionalización ${ }^{(4)}$, y se organiza alrededor de un actor central (Jacques Mabit) que asume una posición ritual de autoridad, que viaja por el mundo para dar a conocer las actividades de la institución. Una clientela internacional se junta en Perú para participar en las prácticas propuestas, mientras que, en varios países, ciertas asociaciones 
proponen la realización de actividades rituales inspiradas en las de Takiwasi. Desde esta perspectiva, la construcción de lugares de culto -que se refieren tanto a la tradición católica y a la historia personal de los principales actores de Takiwasi- aparece como el signo de la aparición y consolidación de una comunidad religiosa que se extiende a escala internacional ${ }^{(4)}$.

Sin negar la relevancia de este enfoque, hay que observar que, sin embargo, la institución no se presenta como un movimiento religioso en sus comunicaciones públicas, sino como una clínica especializada en el tratamiento de la adicción. Además, en oposición a las religiones ayahuasqueras brasileñas (Santo Daime, União do Vegetal, Barquinha, etc.) los principales actores de Takiwasi subrayan que el uso de la ayahuasca no puede ser el fundamento de una religión y prefieren, como lo hemos visto, inscribir sus prácticas como parte de la doctrina católica.

\section{¿Estrategias de legitimación en un contexto de estigmatización?}

Esta ambivalencia puede ser interpretada en función de la porosidad entre los campos religioso y terapéutico que caracteriza las terapias alternativas y las formas contemporáneas de la religiosidad, en particular, las desarrolladas en el marco del movimiento del New Age ${ }^{(27)}$.

Sin embargo, parece significativo que el deseo de ajustar las prácticas rituales de Takiwasi con la doctrina católica aparece cuando la institución y sus principales actores han sido objeto de acusaciones en Francia al describir a Takiwasi como un "grupo sectario $^{\prime \prime(4,28)}$. Entre 2000 y 2003, Jacques Mabit y algunos miembros de las asociaciones francesas vinculadas a Takiwasi fueron procesados después de una denuncia presentada por un padre de familia preocupado por el "cambio de comportamiento" de su hija tratada por psicoterapeutas que ofrecen servicios psicoespirituales inspirados en los de Takiwasi. Esta queja condujo a una investigación que sugirió que la niña habría sido llevada a
Takiwasi por una red de reclutamiento de jóvenes para consumir ayahuasca. Una investigación judicial fue abierta en Francia en 2002 por "abuso fraudulento de debilidad" y violación de la ley de estupefacientes. Entre julio de 2002 y mayo de 2004, los hechos se retransmiten a través de artículos de prensa local y nacional. Considerados como delictivos, se asocia a Takiwasi con una "secta" de "manipulación mental", "posibles suicidios", "abuso" y "fraude". En enero de 2004, Jacques Mabit y psicoterapeutas son objeto de un proceso judicial basado en el cargo de abuso fraudulento de debilidad, lo que conduce finalmente a un no-lugar. La acusación, sin embargo, fue reclasificada como "adquisición, posesión, transporte, suministro, venta de estupefacientes, fraude y abuso de un estado de sugestión cometidos por los líderes de un grupo". Esta acusación, sin embargo, se enfrenta a un nuevo callejón sin salida, ya que la ayahuasca no está clasificada como sustancia prohibida.

Si bien el proceso judicial no dio lugar a una condena de los acusados (la razón principal es que la joven nunca había visitado Takiwasi y nunca había probado la ayahuasca), la movilización de los medios de comunicación tuvo graves consecuencias para la imagen pública de Takiwasi. La misión interministerial de vigilancia y lucha contra las sectas (MIVILUDES) de Francia, cuya función es la de coordinar la acción gubernamental preventiva y represiva contra las sectas, presenta a Takiwasi en dos informes ${ }^{(29,30)}$ como un grupo con estructura típica de "productos derivados del neochamanismo y de sus operaciones en Francia y Europa". El informe denuncia los modos de selección de clientes y el uso de drogas "fuertes" que implican "riesgos de manipulación"(29). Las acusaciones realizadas en 2005 también han dado lugar a la inclusión en la lista francesa de drogas prohibidas a todas las plantas y sustancias que puedan participar en la composición de la ayahuasca. Ese texto, que dota al juez de herramientas capaces de castigar a los grupos que harían uso de la ayahuasca, pone de relieve los riesgos de desestabilización psicológica causada por su consumo 
y su posible uso como una herramienta de "sumisión química", incluso mediante grupos "sectarios". Las asociaciones Liberté du Santo Daime y una asociación vinculada a Takiwasi presentan una solicitud de cancelación de esta decisión, negando la naturaleza tóxica y adictiva de la ayahuasca, así como los riesgos sociales y de salud atribuidos al consumo, y pidiendo el respeto del principio de la libertad de conciencia. Este recurso será, sin embargo, rechazado en 2007 "a la luz de los problemas de salud pública".

Este contexto nos puede ayudar a entender la resistencia de Takiwasi para aparecer públicamente como un grupo religioso en la década de 2000. Además, articular las actividades rituales de la institución con una tradición religiosa establecida (como la Iglesia católica) puede aparecer como una lógica de legitimación de una institución que, al tiempo que tendía a convertirse en movimiento terapéutico-religioso transnacional, era sacudida por acusaciones que comparaban la institución con un "grupo sectario"(4).

El caso Takiwasi ofrece, en este sentido, un ejemplo original de las estrategias de legitimación movilizadas por grupos que hacen uso de la ayahuasca, frente a la política implementada por la mayoría de los estados de los países afectados por el desarollo de estos grupos, la cual consistía mayormente, en los últimos años, en asociar la bebida a una droga prohibida. En efecto, el ingrediente activo que se encuentra generalmente en la decocción (dimetiltriptamina) es en sí mismo clasificado como estupefaciente desde el Convenio sobre Sustancias Sicotrópicas de Viena de 1971, pero no la ayahuasca como tal, por lo que la legislación en esta materia depende de cada Estado.

En numerosos países, grupos que usan la ayahuasca han argumentado a favor de la libertad de religión con el fin de defender el derecho de usar legalmente el brebaje psicotrópico: España ${ }^{(31)}$, Francia ${ }^{(28)}$, Italia $^{(32)}$, Holanda( ${ }^{(33)}$, Alemania ${ }^{(34)}$, EEUU $^{(35,36)}$, Canadá(37).

Estos grupos se reivindican mayormente de dos movimientos religiosos. El primero es el Santo Daime, un movimiento originario de la Amazonia brasileña fundado por Raimondo
Serra Irrineu en 1930. El Ayahuasca, nombrado en este contexto, el "Santo Daime" se utiliza como un sacramento de este culto sincrético que fusiona elementos de inspiración cristiana, indígena y afrobrasileña. El grupo se ha desarrollado ampliamente en las últimas décadas, en especial, en América del Norte y Europa. El segundo es la União do Vegetal. Fundado en Brasil en 1961 por José Gabriel Da Costa, este grupo pone también en el centro de su culto el uso de la ayahuasca (denominado hoasca o vegetal), y tiene ahora grupos en América del Norte y en muchos países europeos $^{(38)}$.

Esta estrategia de legitimación fue exitosa en varios países. Por ejemplo, en EEUU, Santo Daime $^{(36)}$ y União do Vegetal|(35) pidieron una exención de la prohibición de la ayahuasca para respetar la libertad religiosa. El 21 de febrero de 2006, de acuerdo con una decisión del Tribunal Supremo, basada en el Religious Freedom Restoration Act, el uso de la ayahuasca está despenalizado en EEUU en el marco estricto de una práctica religiosa. Así, el Tribunal ha permitido a estos grupos utilizar la ayahuasca en sus rituales. El mismo año también se despenalizó la ayahuasca en Brasil, en el marco de un estricto uso religioso ${ }^{(39)}$. En este contexto, el reconocimiento de estos grupos como organizaciones religiosas por parte de los diferentes estados se está convirtiendo en un tema central, como lo demuestra el ejemplo de España, donde el Santo Daime ${ }^{(31)}$ y la União do Vegetal ${ }^{(40)}$ lograron este estatus gracias a los esfuerzos de sus miembros.

El contexto específico de Francia, marcada por un gran temor a las desviaciones sectarias asociadas a los nuevos movimientos religiosos ${ }^{(26,41)}$ parece desalentar este tipo de estrategias. En este contexto y frente a las acusaciones de la década de 2000, la evocación de la tradición de una religión antigua y reconocida -la Iglesia católica- por parte de los actores de Takiwasi contribuye a proteger la institución de los cargos de abusos sectarios.

Sin embargo, la institución sigue siendo muy discreta en sus comunicaciones públicas sobre el lugar dado al catolicismo, y prefiere comunicar sobre el tema del tratamiento de la adicción. Recientemente, Takiwasi 
también se esfuerza por producir nuevos datos clínicos para certificar los resultados de los métodos utilizados en el tratamiento de la adicción y la psicopatología. De este modo, la movilización del campo médico y científico se puede entender como una estrategia alternativa de legitimación en un contexto de estigmatización del uso de la ayahuasca.

Esta estrategia fue también utilizada en los últimos años por los grupos que hacen uso de la bebida. Las religiones brasileñas ayahuasqueras -Santo Daime, la União do Vegetal, Barquinha- participaron o iniciaron numerosos estudios experimentales, clínicos y epidemiológicos en los últimos años para demostrar la seguridad de la ayahuasca y sus beneficios.

Por ejemplo, grupos del Santo Daime han participado en estudios científicos para establecer la seguridad a largo plazo del uso de la ayahuasca en salud mental ${ }^{(42)}$ y su efecto beneficioso sobre diversas patologías como dolor crónico, cáncer, asma, alergias, depresión, abuso de alcohol, hepatitis, etc. ${ }^{(43)}$. Por su parte, la União do Vegetal trabaja en Brasil con las autoridades del país para que sus prácticas se incorporen a las normas legales y a las exigencias de salud pública ${ }^{(44)}$. En ese contexto, el grupo ha formalizado una serie de recomendaciones que regulan el uso de la ayahuasca al interior de sus grupos para limitar los riesgos de interacciones de drogas y desordenes psicológicos ${ }^{(45)}$.

\section{CONCLUSIÓN}

Al observar la evolución diacrónica de Takiwasi se revela una profunda transformación del cuadro en la que se presentan y se piensan las prácticas ofrecidas por la institución. Como hemos visto, en su fundación, la asociación se constituye en referencia al campo de la medicina, y se apoya sobre la valorización de las "medicinas tradicionales" iniciada por la Organización Mundial de la Salud (OMS) desde la reunión de Alma Ata ${ }^{(46,47)}$. Mientras que en su fundación la asociación se construye en referencia al campo de la medicina, con el tiempo va incluyendo más y más elementos extraídos de la religiosidad occidental contemporánea de inspiración New Age, luego de la tradición católica.

Como hemos sugerido, las mutaciones de la institución pueden ser leídas como efecto de factores sociales (aparición del neochamanismo, acusaciones de "abuso sectario" y prohibición de la ayahuasca por las autoridades francesas), que parecen haber determinado, en parte, estas transformaciones. En este sentido, el caso particular de Takiwasi ilustra los procesos de legitimación que adoptan las instituciones que desarrollan nuevas prácticas híbridas alrededor del uso de ayahuasca frente al estigma de las autoridades públicas en el contexto de la prohibición del brebaje.

A pesar de la diversidad de grupos que usan la ayahuasca, las observaciones anteriores ponen de relieve la necesidad continua de convocar a autoridades establecidas para su legitimación, entre las cuales, la autoridad médica, que tiene el monopolio de la legitimidad del cuidado del cuerpo en las sociedades secularizadas ${ }^{(48)}$, parece central. Desde la época colonial, el patrón terapéutico fue una manera privilegiada de legitimar prácticas rituales que de otro modo estarían prohibidas ${ }^{(49)}$. Los practicantes del chamanismo mestizo peruano, cuya terapéutica es solo uno de sus varios campos de aplicación (favorecer la caza, la pesca, la guerra, brujería, magia de amor, etc.) tienden a destacar el aspecto terapéutico de sus prácticas, para valorizarlas ${ }^{(2)}$, como los practicantes de Santo Daime o de União do Vegetal.

La autoridad religiosa parece también muy importante en la construcción y el mantenimiento de estos grupos que buscan reconocimiento y legitimidad. Hemos visto así que tanto el Santo Daime como el União do Vegetal intentan ser reconocidos oficialmente como una religión por varios Estados y que, por su parte, Takiwasi se inscribe en la tradición de una religión institucionalizada $^{(50)}$ : la Iglesia católica.

En este sentido, el caso especial de Takiwasi pone de relieve tanto la diversidad de las prácticas emergentes alrededor del uso de la ayahuasca y también la permanencia 
del monopolio de las autoridades religiosas y médicas establecidas en la legitimación del cuidado de los cuerpos y de las almas en las sociedades occidentales y poscoloniales. Es significativo, en este sentido, que los prismas religiosos y terapéuticos movilizados por los primeros estudiosos del hecho chamánico $^{(51,52,53)}$, siguen siendo predominantes en el contexto de las reconstrucciones contemporáneas de las prácticas que movilizan la etiqueta "chamánica" alrededor del uso de la ayahuasca.

\section{AGRADECIMIENTOS}

Este artículo se basa en una investigación etnográfica realizada en la alta Amazonía peruana entre 2009 y 2013 como parte de mi investigación doctoral (École des Hautes Études en Sciences Sociales, París). Quisiera dar las gracias a la Fondation Martine Aublet (Museo del Quai Branly), al École des Hautes Études en Sciences Sociales, al Laboratoire d'Anthropologie Sociale y al Institut des Amériques, cuya ayuda financiera hizo posible este estudio. También me gustaría dar las gracias por su consejo y crítica constructiva a versiones anteriores de este texto a Philippe Descola, Patrick Deshayes, Nathalie Luca, Enrique Del Bosque y a los evaluadores anónimos de la revista Salud Colectiva.

\section{REFERENCIAS BIBLIOGRÁFICAS}

1. Losonczy AM, Mesturini Cappo S. Entre I'occidental et l'indien: ethnographie des routes du chamanisme ayahuasquero entre Europe et Amériques. Autrepart. 2010;(56):93-110.

2. Luna LE. Vegetalismo, shamanism among the mestizo population of the Peruvian Amazon. Stockholm: Almqvist \& Wiksell International; 1986.

3. Dupuis D. Pharmacopées indigènes et internationalisation du curanderismo péruvien: le cas de Takiwasi (Haute-Amazonie). In: Gutierrez Choquevilca $\mathrm{AL}$, editor. Cahiers d'anthropologie sociale. No 14. Paris: L'Herne; 2017.

4. Del Bosque E. Le centre Takiwasi: cadres sociaux d'un mouvement religieux-thérapeutique articulé autour de la consommation rituelle d'ayahuasca; memoire de master 2. Paris: E'cole Pratique des Hautes E'tudes; 2011.

5. Caiuby Labate BC. Ayahuasca Mamancuna merci beaucoup: internacionalização e diversificação do vegetalismo ayahuasqueiro peruano. [Teses de Doutorado]. Campinas: Universidade Estadual de Campinas; 2011.

6. Dupuis D. Les murmures de I'ayahuasca: parcours rituel et transmission culturelle à Takiwasi. [Thèse de doctorat]. Paris: École des Hautes Études en Sciences Sociales; 2016.

7. Mabit J. L'hallucination par I'Ayahuasca chez les guérisseurs de la Haute-Amazonie péruvienne [Internet]. Lima: Institut Français d'Etudes Andines; 1989 [citado 14 jul 2017]. Disponible en: https:// tinyurl.com/yb9ht24r.

8. Mabit J. Chamanisme amazonien et toxicomanie: initiation et contre-intiation. Agora-Éthique, Médecine et Société. 1993;(27-28):139-145.

9. Nakazawa Giove RA. La liana de los muertos al rescate de la vida: 7 años de experiencia del Centro Takiwasi. Tarapoto: Takiwasi; 2002.

10. Daniels M. Sombra, yo y espíritu: ensayos de psicología transpersonal. Barcelona: Kairós; 2008.

11. Grof S. LSD psychotherapy. Pomona: Hunter House; 1980.

12. Zinberg NE. Drug, set, and setting: the basis for controlled intoxicant use. New Haven: Yale University Press; 1984. 
13. Vernette J. Le New age. Paris: Presses Universitaires de France; 1992.

14. Champion F. La nébuleuse mystique-ésoterique: orientations psycho-religieuses des courants mystiques et ésoteriques contemporains. In: Champion F, Hervieu-Lèger $\mathrm{D}$, editors. De I'émotion en religion: renouveaux et traditions. Paris: Centurion; 1990. p. 17-69.

15. Wallis RJ. Shamans/neo-shamans: ecstasy, alternative archaeologies and contemporary pagans. London: Routledge; 2003.

16. Riera F. Visions de Takiwasi: une approche anthropologique d'un métissage thérapeutique; mémoire de Master 2 Dynamiques Identitaires option Ethnologie. Brest: Université de Bretagne Occidentale; 2011.

17. Taymans $\mathrm{O}, \mathrm{D}^{\prime}$ Olivier I. Takiwasi, la maison qui chante [Internet]. Belgique: SilenceRadio; 2005 [citado 14 jul 2017]. Disponible en: https:// tinyurl.com/yakk962x.

18. Bernardi A, Kelner G, Sibra M, Torikian G. L'ayahuasca, le serpent et moi [Video en internet]. Artline Films, France 5, productores; 2004 [citado 14 jul 2017]. Disponible en: https://tinyurl.com/ ybbzh4mg.

19. Cheyssial JC. L'esprit de I'ayahuasca [Video en internet]. La Luna Productions, Réseau France outre-mer, Latitude; 2002 [citado 14 jul 2017]. Disponible en: https://tinyurl.com/yclqtoh2.

20. Apffel-Marglin F. Itinerary and testimony of Dr. Jacques Mabit, physician and shaman. InterCulture. 2007;(152):25-46.

21. Lévi-Strauss C. Anthropologie structurale. Paris: Plon; 1958.

22. Weber M. Économie et société. Paris: Pocket; 1971.

23. Mary A. Les anthropologues et la religion. Paris: Presses Universitaires de France; 2010.

24. Tournyol Du Clos JC. Peut-on se libérer des esprits impurs?; un guide pratique vers la délivrance. Beyrouth: Éditions de l'Archistratège; 2007.

25. Halloy A, Dupuis D. Les racines émotionnelles de la possession religieuse: une ethnographie comparative. Intellectica. 2017;(67):301-325.

26. Luca N. Individus et pouvoirs face aux sectes. Paris: Armand Colin; 2008.

27. Masse R, Benoist J. Convocations thérapeutiques du sacré. Paris: Karthala; 2002.
28. Bourgogne G. One hundred days of ayahuasca in France: the story of a legal decision. In: Caiuby Labate $\mathrm{BC}$, Jungaberle $\mathrm{H}$, editores. The internationalization of ayahuasca. Zurich: Lit Verlag; 2011. p. 353-363.

29. Mission Interministérielle de Vigilance et de Lutte Contre les Dérives Sectaires. Rapport annuel 2005: la protection des mineurs et la sécurité des entreprises face à l'emprise sectaire; risques de dérives dans I'aide humanitaire [Internet]. Paris: MIVILUDES; 2005 [citado 14 jul 2017]. Disponible en: https://tinyurl.com/yd9a87g4.

30. Mission Interministérielle de Vigilance et de Lutte Contre les Dérives Sectaires. Rapport annuel 2009: les mineurs et le risque sectaire. Paris: MIVILUDES; 2009 [citado 14 jul 2017]. Disponible en: https://tinyurl.com/yctfqcs9.

31. López-Pavillard S, De Las Casas D. Santo Daime in Spain: a religion with a psychoactive sacrament. In: Caiuby Labate BC, Jungaberle $\mathrm{H}$, editores. The internationalization of ayahuasca. Zurich: Lit Verlag; 2011. p. 365-374.

32. Menozzi W. The Santo Daime legal case in Italy. In: Caiuby Labate BC, Jungaberle $\mathrm{H}$, editores. The internationalization of ayahuasca. Zurich: Lit Verlag; 2011. p. 379-388.

33. Van den Plas A. Ayahuasca under international law: the Santo Daime churches in the Netherlands. In: Caiuby Labate BC, Jungaberle H, editores. The internationalization of ayahuasca. Zurich: Lit Verlag; 2011. p. 327-338.

34. Rohde SA, Sander H. The development of the Santo Daime legal situation in Germany. In: Caiuby Labate BC, Jungaberle $\mathrm{H}$, editores. The internationalization of ayahuasca. Zurich: Lit Verlag; 2011. p. 339-351.

35. Bronfman J. The legal case of the União do Vegetal vs the Government of the United States. In: Caiuby Labate BC, Jungaberle $\mathrm{H}$, editores. The internationalization of ayahuasca. Zurich: Lit Verlag; 2011. p. 287-300.

36. Haber R. The Santo Daime road to seeking religious freedom in the USA. In: Caiuby Labate BC, Jungaberle $\mathrm{H}$, editores. The internationalization of ayahuasca. Zurich: Lit Verlag; 2011. p. 301-317.

37. Tupper KW. Ayahuasca in Canada: cultural phenomenon and policy isssue. In: Caiuby Labate $B C$, Jungaberle $H$, editores. The internationalization of ayahuasca. Zurich: Lit Verlag; 2011. p. 319-325.

38. Hanegraaff WJ. Ayahuasca groups and networks in the Netherlands: a challenge to the study 
of contemporary religion. In: Caiuby Labate BC, Jungaberle $\mathrm{H}$, editors. The internationalization of ayahuasca. Zurich: Lit Verlag; 2011. p. 85-103.

39. Boiteux L. The new brazilian law on drugs and the religious uses of ayahuasca: legal and anthropological aspects. In: Caiuby Labate BC, Jungaberle $\mathrm{H}$, editores. The internationalization of ayahuasca. Zurich: Lit Verlag; 2011. p. 263-275.

40. Marin Prades JV. Legal recognition of the União do Vegetal in Spain. In: Caiuby Labate BC, Jungaberle $\mathrm{H}$, editores. The internationalization of ayahuasca. Zurich: Lit Verlag; 2011. p. 375-378.

41. Esquerre A. La manipulation mentale: sociologie des sectes en France. Paris: Fayard; 2009.

42. Bouso JC, Fábregas JM, González D, Fondevila S, Fernández X, Cutchet M, Alcázar MA. Long-term effects of the ritual use of ayahuasca on mental health. In: Caiuby Labate BC, Jungaberle $\mathrm{H}$, editores. The internationalization of ayahuasca. Zurich: Lit Verlag; 2011. p. 167-184.

43. Schmid JT. Ayahuasca healing: a qualitative study about fifteen european people handling their diseases. In: Caiuby Labate BC, Jungaberle $\mathrm{H}$, editors. The internationalization of ayahuasca. Zurich: Lit Verlag; 2011. p. 245-259.

44. Soares ELC, Moura CP. Development and organizational goals of the União do Vegetal as a brazilian and international religious group. In: Caiuby Labate $\mathrm{BC}$, Jungaberle $\mathrm{H}$, editores. The internationalization of ayahuasca. Zurich: Lit Verlag; 2011. p. 277-286.

45. Lima FAS, Tófoli LF. An epidemiological surveillance system by the UDV: mental health recommendations concerning the religious use of hoasca. In: Caiuby Labate BC, Jungaberle $\mathrm{H}$, editores. The internationalization of ayahuasca. Zurich: Lit Verlag; 2011. p. 185-199.

46. World Health Organization. The promotion and development of traditional medicine: report of a WHO meeting [Internet]. Geneva; 1977 [citado 14 jul 2017]. Disponible en: https://tinyurl. com/y967qkzd.

47. Organisation Mondiale de la Santé. Déclaration d'Alma-Ata: conférence internationale sur les soins de santé primaires [Internet]. Alma-Ata; 1978 [citado 14 jul 2017]. Disponible en: https:// tinyurl.com/y8y94eo7.

48. Laplantine F. Anthropologie de la maladie: étude ethnologique des systèmes de représentations étiologiques et thérapeutiques dans la société occidentale contemporaine. Paris: Payot. 1993.

49. Olivier De Sardan JP. Possession, affliction et folie: les ruses de la thérapisation. L'Homme. 1994;34(131):7-27.

50. Hervieu-Leger D. La religion pour mémoire. Paris: Les Editions du Cerf; 1993.

51. Mihajlovskij VM. Shamanstvo: sravnitel'noetnograficeskie ocerki. Moskva: Izvestiya Imperatorskago Obscestva Ljubitelej Estestvoznanija, Antropologii i Etnografii; 1892.

52. Ohlmarks A. Studien zum problem des schamanismus. Kopenhagen: Lund; 1939.

53. Eliade M. Shamanism: archaic techniques of ecstasy. Princeton: Princeton University Press; 1964. 